\title{
Enhancing MANET by Balanced and Energy Efficient Multipath Routing with Robust Transmission Mechanism with Using FF-AOMDV
}

\author{
Naif D. Alotaibi, Elyas I. Assiri \\ Electrical and Computer Engineering Department, King Abdulaziz University (KAU), Jeddah, Saudi Arabia \\ Email: elyasassiri@gmail.com
}

How to cite this paper: Alotaibi, N.D. and Assiri, E.I. (2021) Enhancing MANET by Balanced and Energy Efficient Multipath Routing with Robust Transmission Mechanism with Using FF-AOMDV. Communications and Network, 13, 131-142. https://doi.org/10.4236/cn.2021.134010

Received: September 2, 2021

Accepted: October 31, 2021

Published: November 3, 2021

Copyright $\odot 2021$ by author(s) and Scientific Research Publishing Inc. This work is licensed under the Creative Commons Attribution International License (CC BY 4.0).

http://creativecommons.org/licenses/by/4.0/

\begin{abstract}
Networking is one of the major areas in which technology is running. Networking plays a vital role in communications for the past few decades. Nodes are the important connections in the network. One of the main parameters considered in the node is power. Power is limited for the nodes in MANET, which is used for some operations where the node energy is not exhausted. Power obligations always affect the systems which ultimately affects the connectivity in the network. Mobility and congestion of nodes are also affected by power problems which simultaneously have some effects like link failures, packet losses which lead some problems in QoS performance of the protocol. This research combines BEMRT (Balanced and Energy Efficient Multipath Routing) with Robust Transmission in MANET. This combination will help the network to survive the problems discussed above which depend on the route discovery mechanism FF-AOMDV. The proposed algorithm will increase the connectivity from end to end and also minimizes the errors at all. Neighbor node selection method is considered with efficient energy which generates multiple paths from source to destination. A stable path is found by the node with effective load balancing. The mechanism is simulated and the protocol FF-AOMDV is compared with AOMDV and AOMRLM with concerning PDR (packet delivery ratios), routing overhead, delay, energy consumption, network lifetime, and throughput.
\end{abstract}

\section{Keywords}

BEMRT, MANET, PDR, Networking 


\section{Introduction}

Data communication and networks are the important field where has driven and impacts many areas. All the embedded systems or other data communication systems purely depended on the internetwork or computer networks. Any network contains a lot of nodes, so the nodes are the basic of network architecture. The nodes can be send or receive any information for the network system. All the nodes communicate by the links with each other [1]. The links are connected by the channels also named as communication channels. These channels can be telecommunication, optical, wireless, etc. One of the easy examples is internet where many systems are interconnected together where there will be some slave systems and some master systems [2]. For all networks, it should follow some rules as given

Performance: it is calculated as

- Transit time: the time taken to pass the information from one system to another system.

- Response time: The time elapsed between enquiry and response. Some other ways to calculate the performance:

1) Software effectiveness

2) Users subscribed

3) Hardware interference

\section{Reliability}

It shows how reliable the network is. It is measured by the number of faults in the network. Less the number of errors in the network, more reliable the network is designed.

\section{Security}

Security means protection by non-subscribed users. No other users can affect the network. Security should give for all the nodes and channels sine data can be communicated through any channel and any node [3]. So, in the network, security plays an important role.

\section{Research Questions}

- What are the important nodes?

- What are the techniques which are used for improving the parameters?

- What methods accomplish the promising results in terms of power related problems?

- What is the best path for transferring the information with high quality and low cost?

\section{Objectives}

- To perform proposed parameters in terms of packet delivery ratio, normalized routing overhead, throughput, and average end to end delay.

- To obtain motion parameters i.e. velocity, direction of the nodes. Based on these parameters, the network selects the path to transmit the data packets between the nodes.

- To take care of the battery level of the node scan in the network. This results in network's good throughput and high efficiency. 


\section{Block Description}

Basic communication model communicates the information from one place to another. Source generates the information which is transmitted. Transmission system is a network which carries the information to a particular receiver [4]. The receiver receives the information and sends to the destination. The whole process is shown in Figure 1. The transmission network can be wired or wireless. Communication can be local or remote [5]. Local is commutation inside a particular area or building. Remote is commutation over some particular distance like cities.

\section{MANET}

Due to the design of many costless wireless devices, there is exponential growth in the mobile computing area. Therefore, huge research is going on in networks. MANET is a mobile ad hoc network where commination is done within its nodes only. Devices outside its transmission range need to switch to other devices [6]. To install the base station autonomous systems the mobile hosts are used. Single hop cellular network model is used for this installation. Communicating two mobile nodes is relayed on fixed base stations. Nodes are free and can move anywhere in MANET.

MANET networks are peer-to peer multi-hop mobile wireless networks, store and forward manner is used for packet transformation as shown in Figure 2. The expired topology can also easily update. There are symmetric and asymmetric links in this MANET [7].

MANET does not require any fixed infrastructure. These are designed by a self-organizing principle [8]. Multi hop mechanism is used where routing is done between mobile devices. The devices can act as s router as well. The MANET gives best quality in terms of bandwidth, quality of transmission, enhancement, and power control.

\section{Existed System}

There are many existing systems available like SUPERMAN, UAV and VAN.

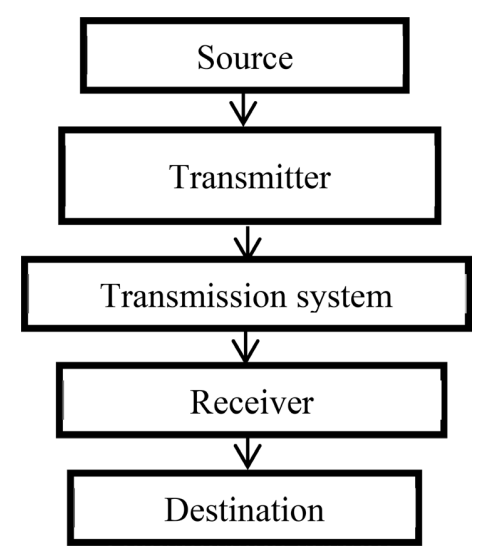

Figure 1. Block diagram. 
Superman process is one of the mostly used processes which are shown in Figure 3. End to end communication parameter is available in superman process [9]. It is processed with three different layers as mentioned

1) Transport layer

2) Network layer

3) Data link layer

Transport protocol and data link protocol is used in transport and data link layer. Security tale and routing table is considered in network layer [10]. The data is protected confidentially in this process.

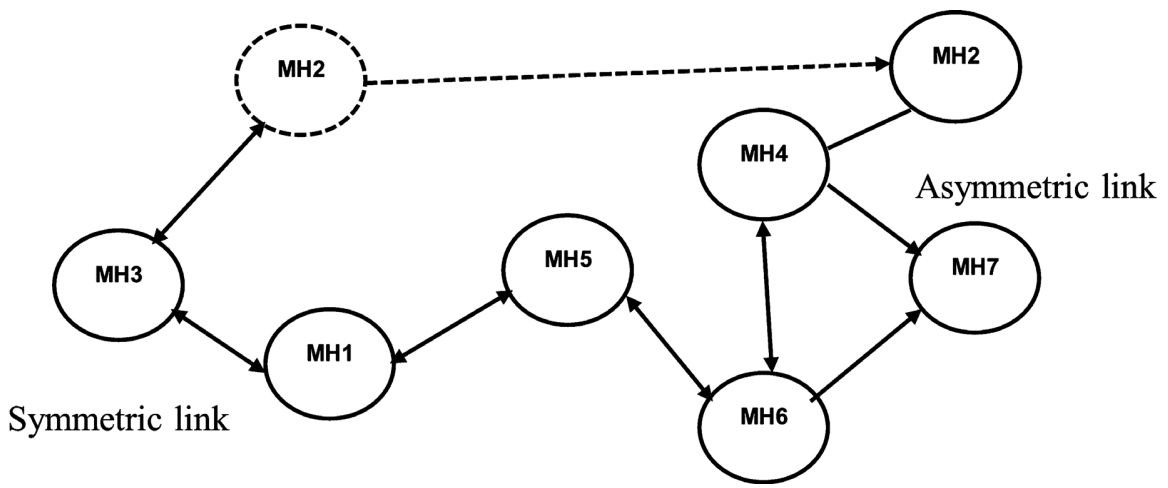

Figure 2. MANET Flow diagram.

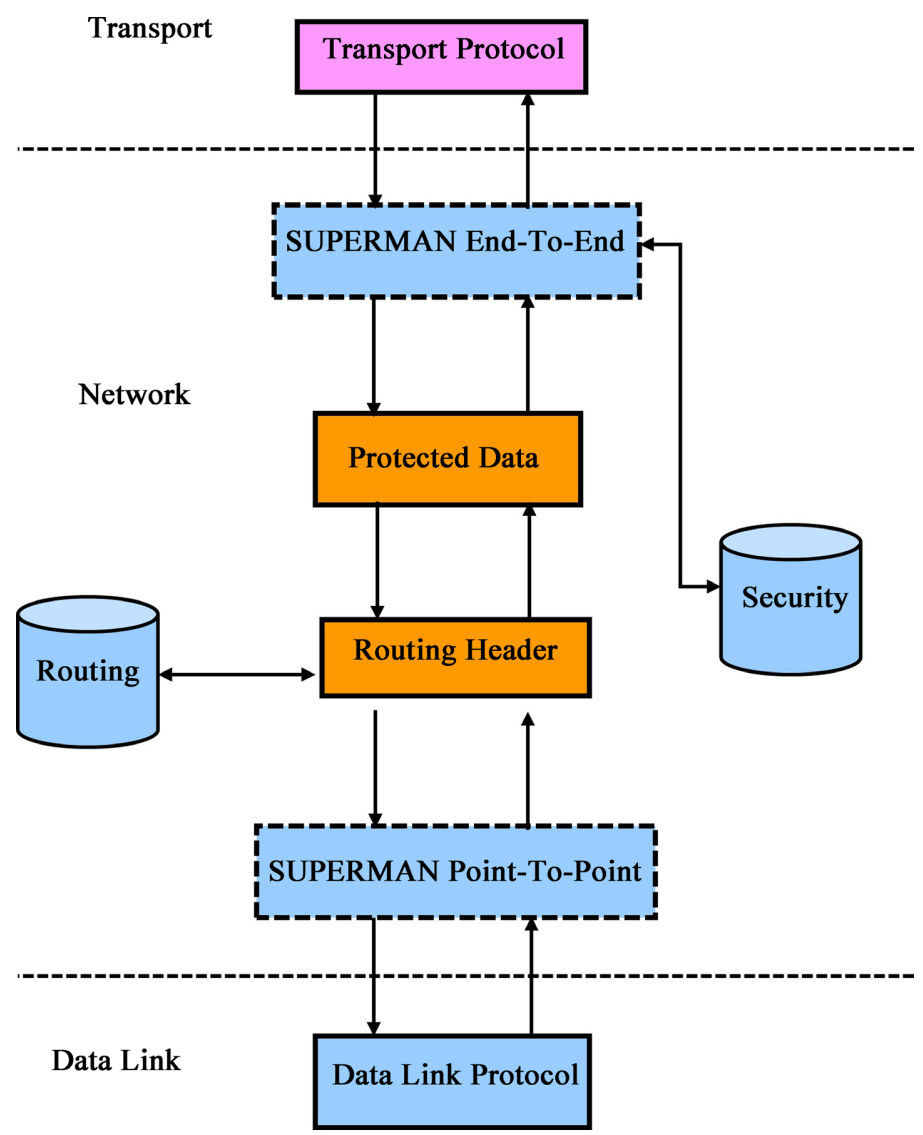

Figure 3. Superman process. 


\section{Problems in existing work:}

Network is always where some problem can be identified. Power obligations are one of the major problems which always affect the systems which ultimately affects the connectivity in the network [8]. Mobility and congestion of nodes is also affected by power problems which simultaneously have some effects like link failures, packet loses which leads some to some problems in QoS performance of the protocol. In a normal scenario, when a RREQ is broadcasted by a source node, more than one route to the destination will be found and the data packets will be forwarded through these routes without knowing the routes' quality (nodes power in these rotes). There is no best performance, reliability and security in the mechanisms which are already generated and operated.

\section{Methodology Proposed}

This research combined BEMRT with Robust Transmission in MANET. This combination will reside the problems which occurs in ad hoc networks. This combination minimizes the errors at all the levels. Energy efficient neighbor node mechanism sets multi path from source to destination. Stable path is created between source and destination. The output is simulated with respect to packet delivery ratio, throughput, routing overhead and average end to end delay. A new QoS with balanced Energy efficiency depended on route discovery mechanism of FF-AOMDV is implemented. "Fitness Function Ad Hoc on Demand Multipath Distance Vector" is used for good efficiency. Stable transmission is done by considering various factors like strength in the signal, length of the queue and the delay. Multipath is just defined as source and destination have multiple routes. Multipath helps that even if one route is failure and has some problem the device can choose the other path. Multi path decreases the faults in the transmission of data. Link state routing algorithms or vector distances are used for routing the protocols. These algorithms need periodic routing advertisements. Considering the overall topology in the network every individual router is broadcasted with all the other routers. It measures which is the shorter distance by considering many factors required for networking. Mainly link state routing is done in this process which improves the networking speed and minimizes the cost and power. Ultimately the mechanism is simulated and the protocol FF-AOMDV is compared with AOMDV and AOMRLM with respect to PDR (packet delivery ratios), routing overhead, delay, energy consumption, network life time and throughput.

\section{Architecture of the System}

The architecture of the proposed system is shown in Figure 4. Firstly, the route needs to be discovered. Next the neighbor and links are selected. Balanced multi path algorithm is used for routing. Selection is done based on genetic. Finally delay awareness and route maintenance. 


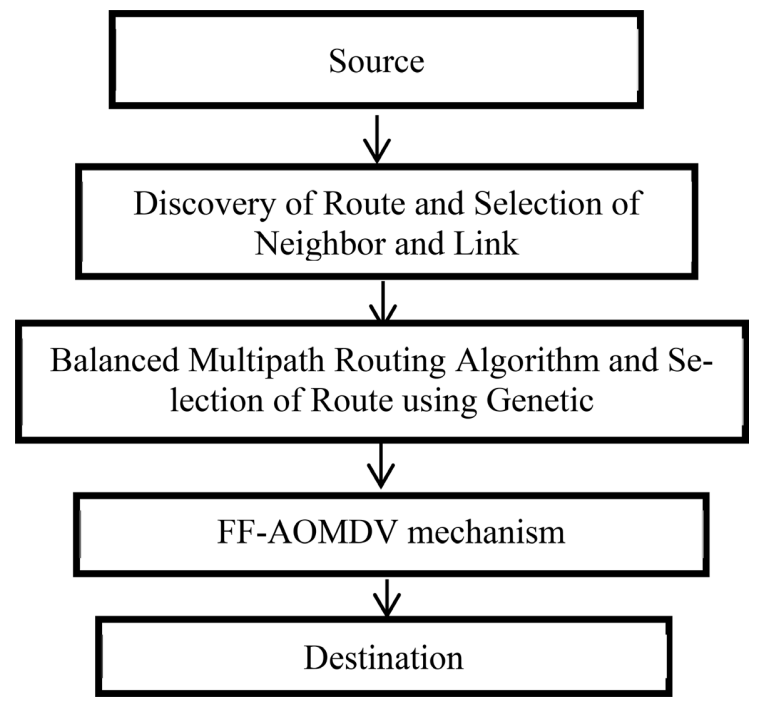

Figure 4. Architecture of the proposed system.

\subsection{Initialization}

The initialization is the first process in which neighour selection and link estimation is done.

\section{Neighbor selection:}

Every sensor has three parameters (sensorID, groupID, $\lambda$ )

$\lambda$ is the capacity of sensor depends on uplink and downlink bandwidths. This is calculated as given in equation 1.

$$
\lambda=\frac{\left(B W_{u p}+B W_{\text {down }}\right) \cdot \text { Available }_{\mathrm{Con}} \cdot L+K_{2}}{\operatorname{Max}_{\mathrm{Con}}} \cdot \sqrt{1-\frac{E^{2}}{K_{1}}} \cdots
$$

Available ${ }_{\mathrm{Con}}$-available number of links

$\operatorname{Max}_{\text {Con }}$-number of maximum links

$B W_{u p}$-uplink frequency

$B W_{\text {Down }}$-Downlink frequency

The higher the $\lambda$ parameter the perfect sensor could be designed. By considering this factor best sensors are designed.

\section{Link Estimation}

WSN is an integrated area where packets of data are generated which is routed perfectly especially in real time operating systems. The link quality is estimated by periodic beacon exchange. The link quality is always affected by high traffic. Queue length estimation is considered for efficient energy systems.

\subsection{Balanced Multipath Routing}

Generally, one way of balancing is by using complex routing, but this method is not useful for all the applications, so another method is network load balancing. This technique is best in terms of emails and web etc. this balancing method provides redundancy in the network. This method balances the Inbound traffic and Outbound traffic. This balanced multipath routing routes the data from 
bridging. While transportation at least one intermediate node is considered for higher efficiency.

\subsection{Genetic Based Route Selection}

Robust based route selection is considered with genetic classification. (GA) genetic algorithms are evaluated from (EA) evolutionary algorithms. GA precisely gives solutions with high quality to optimize and search the problems. GA is always depended on bio-inspired operators like mutation, crossover and selection

\subsection{FF-AOMDV Mechanism}

Delay aware Routing in which route discovery mechanism of FF-AOMDV is modified to include multiple metrics of signal strength, queue length, drain rate and the delay. Route maintenance is the way how the route is maintained from $\mathrm{A}$ to $\mathrm{E}$. A is the source where the packet is transmitted to $\mathrm{E}$ through $\mathrm{B}, \mathrm{C}, \mathrm{D}$ nodes. $\mathrm{B}$ is responsible for $\mathrm{C}$ and $\mathrm{C}$ for $\mathrm{D}$ and $\mathrm{D}$ for $\mathrm{E}$. The FF-AOMDV is an optimization method that uses the fitness function, in this method we search for two parameters in order to select the optimum route is; the route distance and energy level of the route to transfer the data to the destination more efficiently by prolonging the network lifetime and consuming less energy.

\subsection{Advantages}

- Parameters are obtained in terms of PDR, NRO, TDR and EED.

- Motion parameters are also generated like direction, velocity of nodes.

- The selection of path in the network is easy with the generated parameters.

- Best path can be established with the generated parameters.

- Scan of the battery level at the nodes can be done perfectly, due to this high efficiency and throughput can be observed at the results.

- This proposed algorithm has lowest energy consumption.

- The node at the source has 3 types of information.

- All node energy levels are known.

- All route ranges are known.

- While discovering the route the energy consumed is known.

- Easily the route which needs less energy can be found by the minimum distance route and maximum energy level route.

- Later the energy needed is calculated by packets send at maximum energy levels.

- This proposed technique gives multipath routing, which finds a new route from source to destination when general route is failed.

- Alternative new route is selected from the route table, where this route has minimum distance with maximum energy consumption.

\section{Results and Discussions}

The simulation is done in "NS-2.3.4" software which is a network simulator 
where design language is "TCL" (Tool Commanding Language) with "C++" protocol design. Virtual machine is "VM workstation pro -12.1". Many simulations are done to generate precise performance of WSN (wireless sensor network). Random distribution is done for all the nodes. Location of the sink is also given randomly. Various estimations are calculated about the performance of router. Different node and request are taken, and average energy is calculated in three detections.

Long delivery latency is observed with the increase in Ns which ultimately increases length of the routing path. Some of the observations like delay ratio, energy consumption ratio, packet delivery ratio and throughput ratio and comparisons of various protocols graphs are shown below

\subsection{Delay Ratio}

End-to-end Delay is the time delay experienced by the source node while transmitting a report message to the sink. From results of the simulation, we can see the comparing between previous and present process of delay ratio, here red line mention existing delay ratio and green line is proposed delay ratio, in our proposed work reduces the delay compared to existing process system as shown in Figure 5.

Maximum delay time is $900.00 \times 10^{3}$ within all numbers of nodes in existing

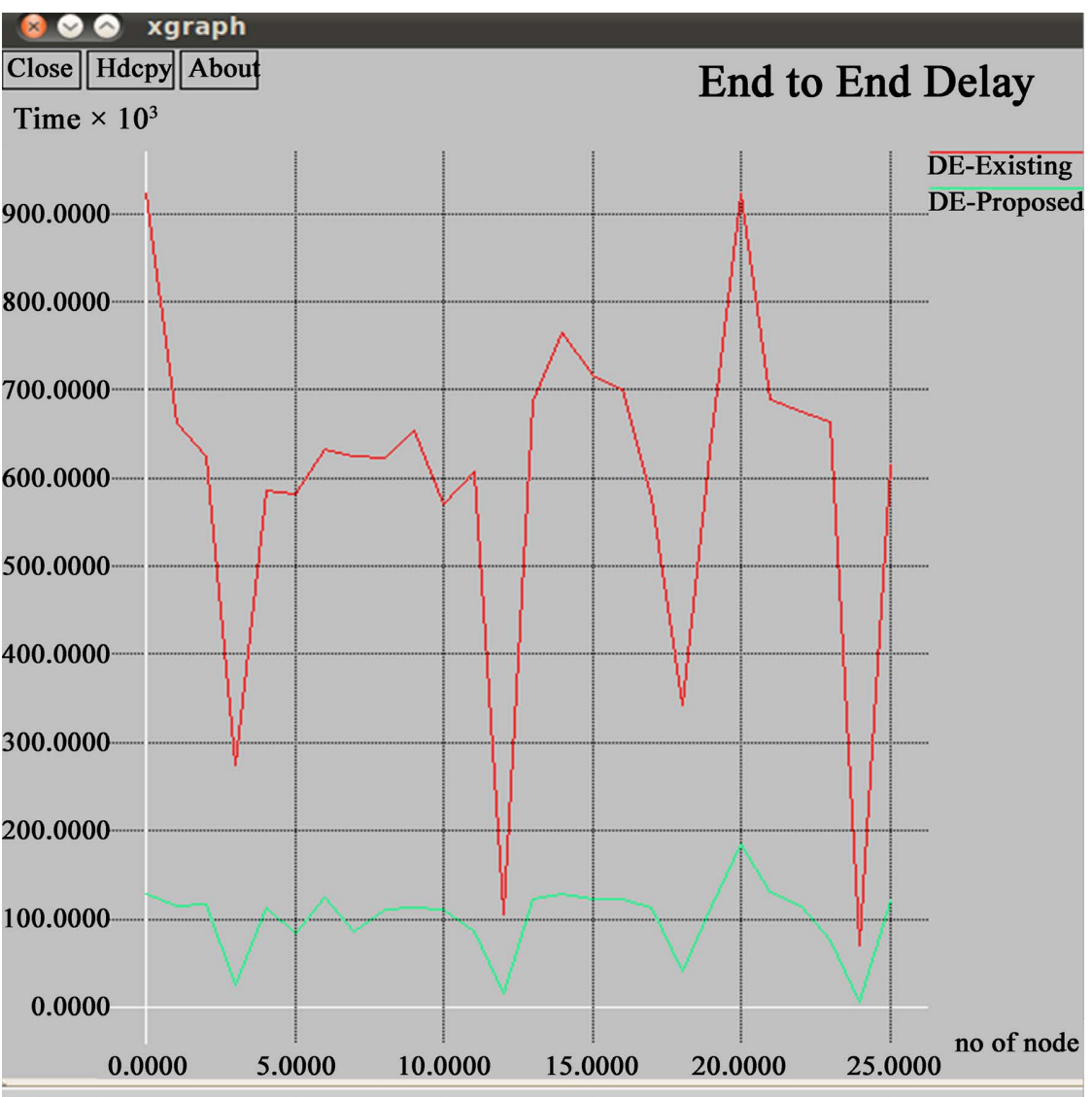

Figure 5. Delay ratio. 
system. And in proposed system the delay time ration reduces to less than 200.00 $\times 10^{3}$ within all numbers of nodes.

\subsection{Energy Consumption Ratio}

Energy Consumption ratio are measuring the mean value of the usage energy of all alive sensor nodes when simulation terminates. In this work compare previous and present process of energy Consumption rate, here green line mention proposed energy ratio and red line is existing energy ratio. Our proposed work reduces consumption of energy compared to existing process as shown in Figure 6.

The time of energy consumption reduced in proposed system, and it is vacillating between $80.00 \times 10^{3}$ and $100.00 \times 10^{3}$ but in proposed system it is decreasing from $70.00 \times 10^{3}$ to less than $50.00 \times 10^{3}$.

\subsection{Packet Delivery Ratio}

In this work compare previous and present process of Packet delivery, here green line mention proposed ratio and red line is existing packet ratio. In our proposed work improves Packet delivery rate compared to existing process as shown in Figure 7.

From Figure 7, we can see the difference between proposed and existing packet ratio. Packet delivery improved from $70.00 \times 10^{3}$ to more than $58.00 \times 10^{3}$ and it keeps more better when the numbers of nodes increasing.

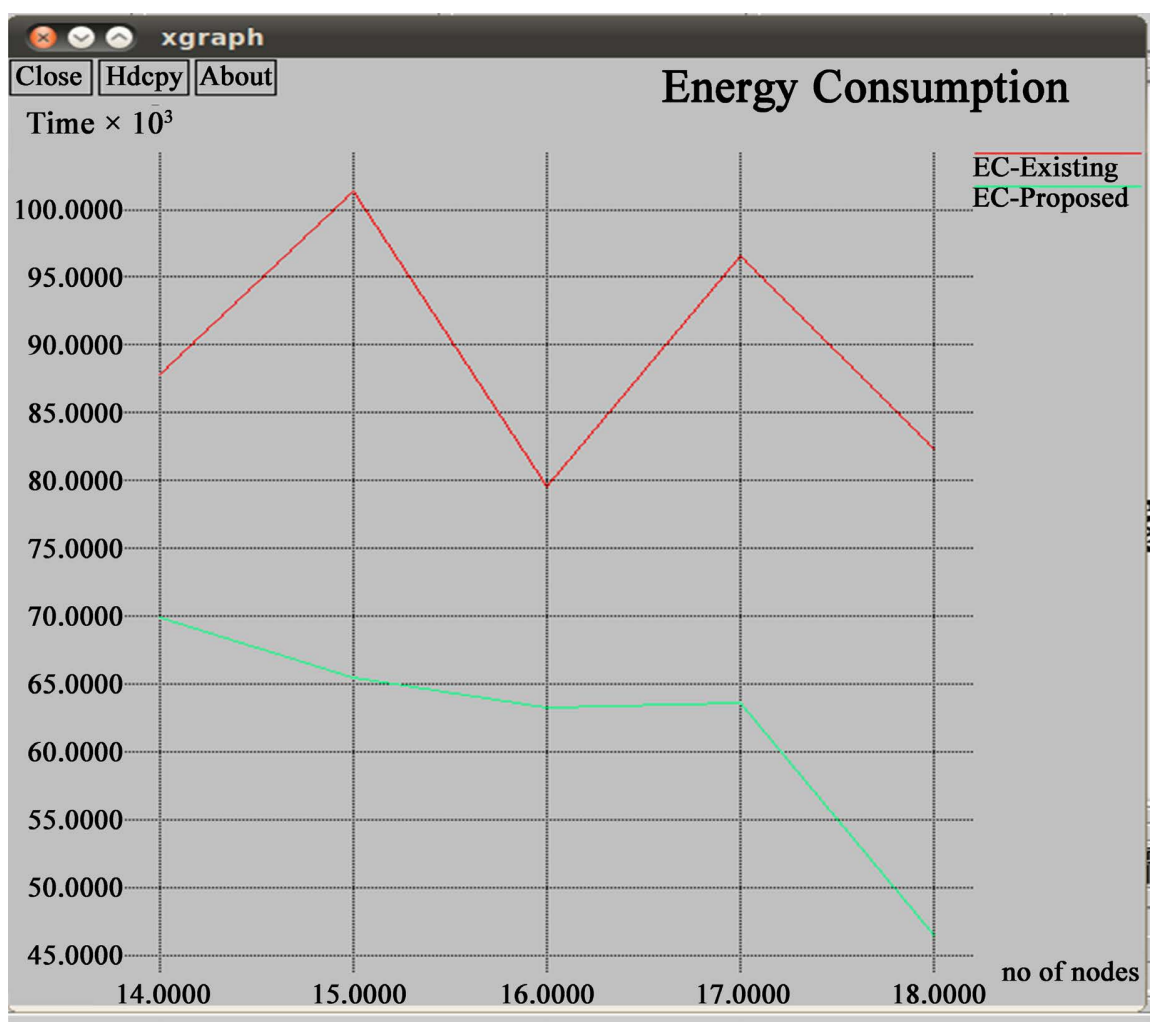

Figure 6. Energy consumption ratio. 


\subsection{Average Throughput Ratio}

In this work comparing the previous and present process of Throughput ratio, here green line mention proposed, red line is existing ratio, in our proposed work improved Throughput ratio compared to existing and previous process as shown in Figure 8.

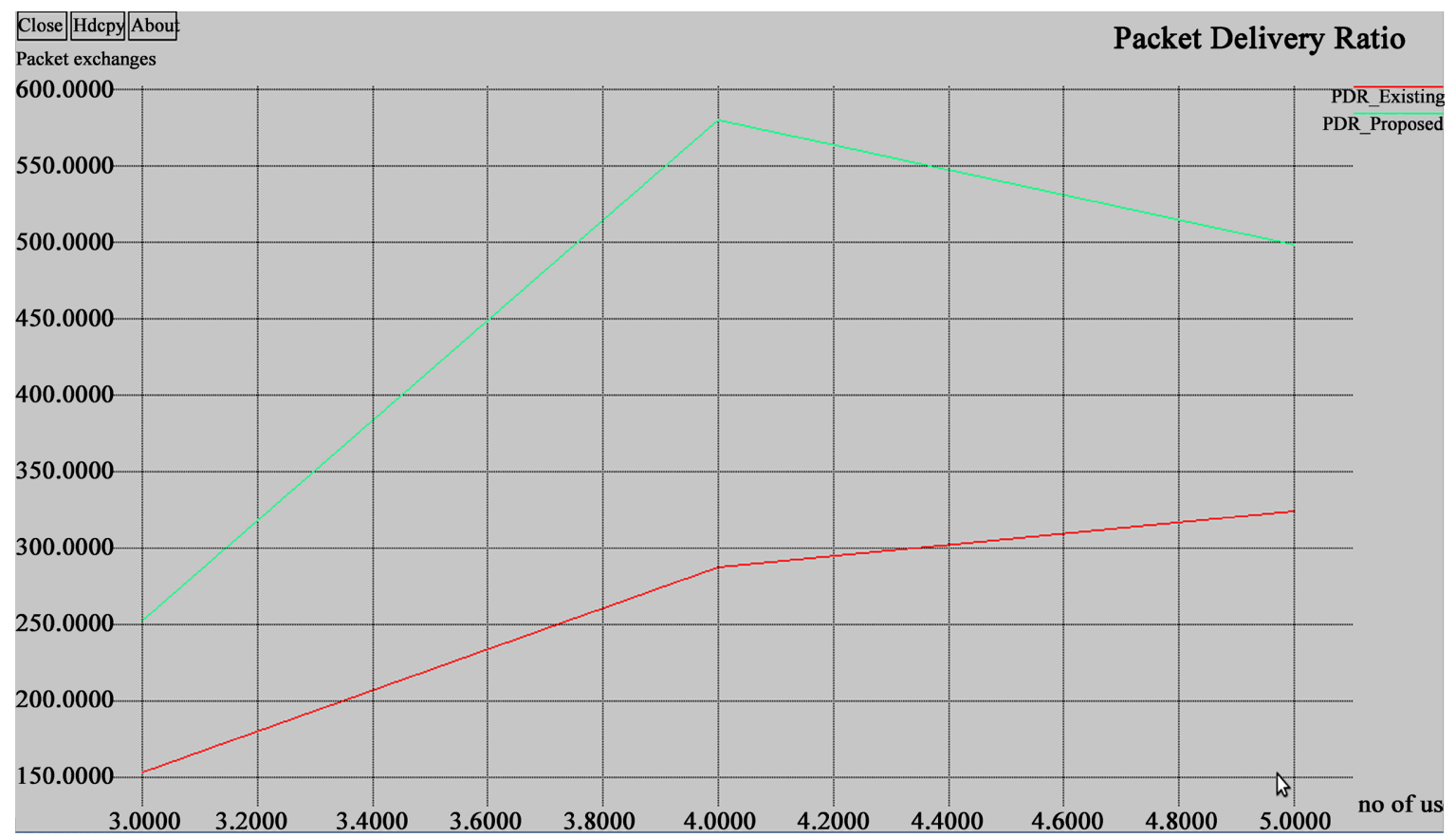

Figure 7. Packet delivery ratio.

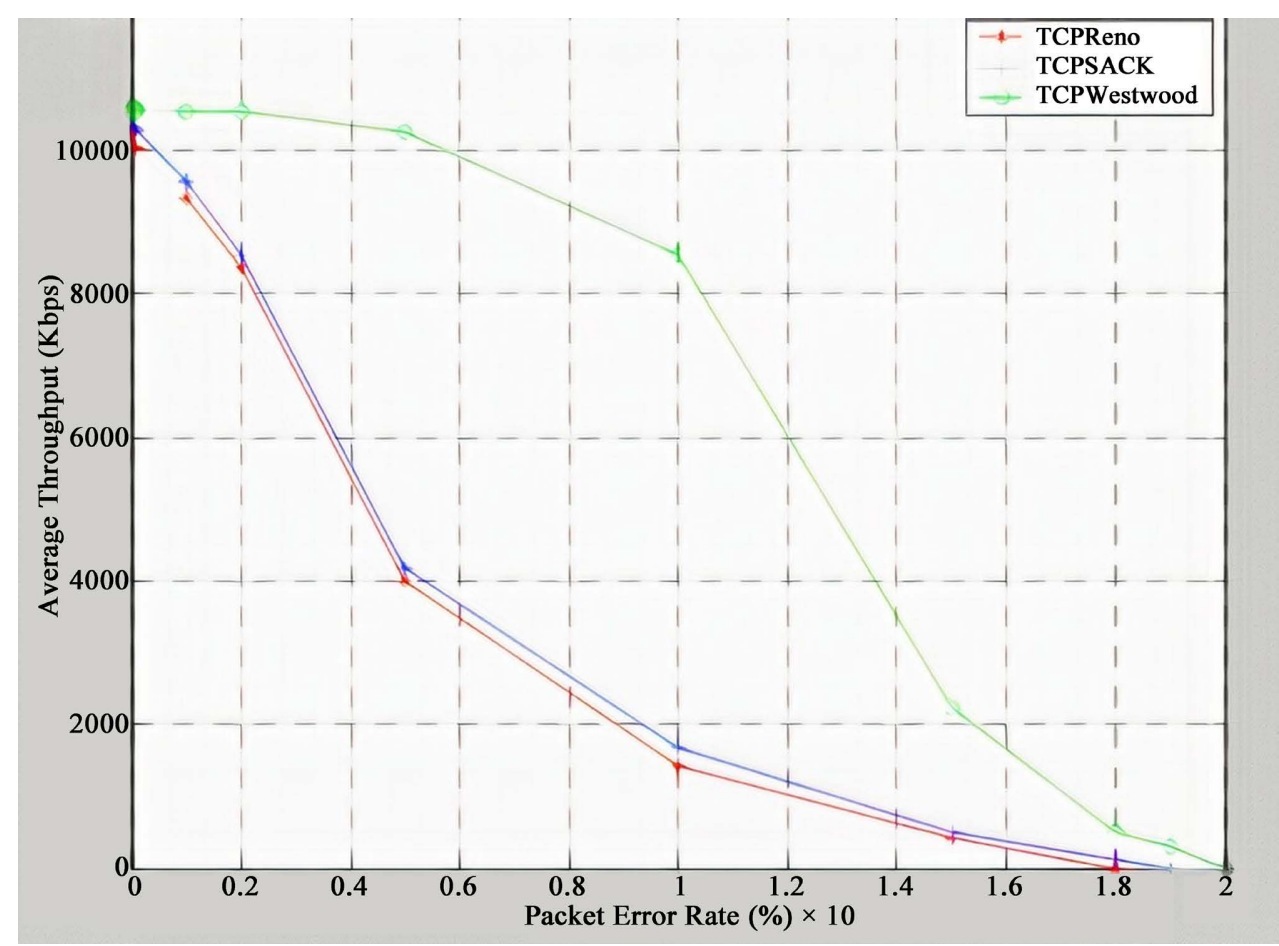

Figure 8. Average throughput ratio. 


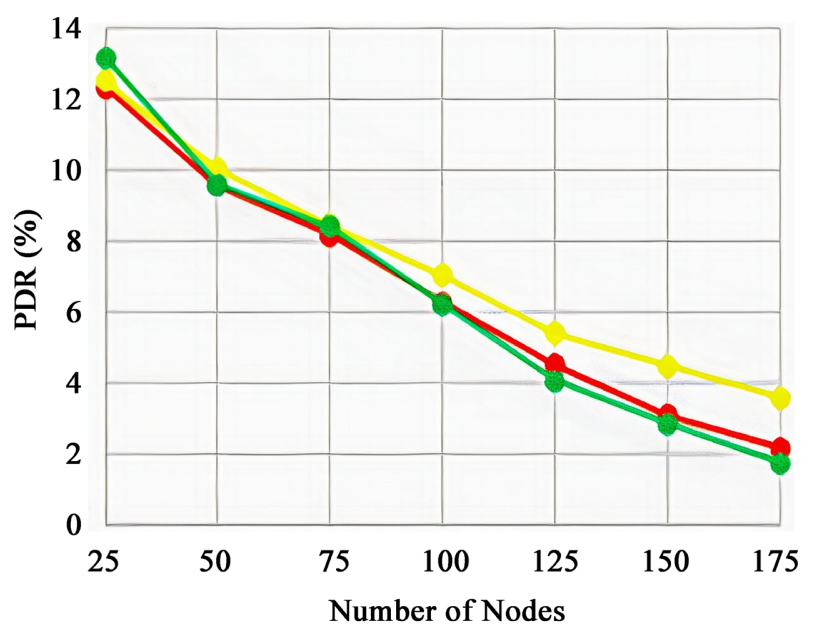

Figure 9. Packet Delivery ratio of FF-AOMDV, AOMDV, AOMRLM.

Packet delivery ratio comparison for FF-AOMDV, AODV and AOMRLM is shown in Figure 9. Yellow line indicates FF-AOMDV, green indicates AOMDV and Red indicate AOMRLM. These three protocols can be used with BEMRT mechanism.

\section{Conclusion}

This research proposed an effective method Balanced and Energy Efficient Multipath Routing with Robust Transmission in MANET. It is shown that the limitations are crossed in MANET. The comparison of FFAOMDV, AAOMDV and AOMRLM is done and proved that FFAOMDV is the best protocol used for this mechanism. This research also maximizes the connections and minimizes the errors in all the levels. Multipath is generated from source to destination. The balancing node is done perfectly and also the stable path is founded easily. The delay is also reduced. It also proves that energy consumption is low. Packet delivery ratios and average throughput ratios are improved.

\section{Conflicts of Interest}

The authors declare no conflicts of interest regarding the publication of this paper.

\section{References}

[1] Kiran, P.S. (2009) Protocol Architecture for Mobile ad Hoc Networks. IEEE International Advance Computing Conference (IACC2009), Patiala, 6-7 March 2009, 1-6.

[2] Wang, W., Yang, B., Takahashi, O., Jiang, X. and Shen, S. (2018) On the Packet Delivery Delay Study for Three Dimensional Mobile Ad Hoc Networks. Ad Hoc Network, 69, 38-48. https://doi.org/10.1016/j.adhoc.2017.10.004

[3] Yang, D., Xia, H., Xu, E., Jing, D. and Zhang, H. (2018) Energy-Balanced Routing Algorithm Based on Ant Colony Optimization for Mobile Ad Hoc Networks. Sensors, 18, 3657. https://doi.org/10.3390/s18113657

[4] Smith, D., Wetherall, J., Woodhead, S. and Adekunle, A. (2014) A Cluster-Based 
Approach to Consensus Based Distributed Task Allocation. 22nd Euromicro International Conference on Parallel, Distributed, and Network-Based Processing, Turin, 12-14 February 2014, 428-431. https://doi.org/10.1109/PDP.2014.87

[5] Chakeres, I.D. and Belding-Royer, E.M. (2004) Aodv Routing Protocol Implementation Design. 24th International Conference on Distributed Computing Systems Workshops, Tokyo, 23-24 March 2004, 698-703. https://doi.org/10.1109/ICDCSW.2004.1284108

[6] Qu, W. and Wang, X. (2017) An Energy-Saving Routing Strategy Based on Ant Colony Optimization in Wireless Sensor Networks. Proceedings of the International Conference in Swarm Intelligence, 10385, 277-284.

https://doi.org/10.1007/978-3-319-61824-1 30

[7] Hyland, M., Mullins, B.E., Baldwin, R.O. and Temple, M.A. (2007) Simulation-Based Performance Evaluation of Mobile Ad Hoc Routing Protocols in a Swarm of Unmanned Aerial Vehicles. 21 st International Conference on Advanced Information Networking and Applications Workshops, AINAW07, 2, 249-256. https://doi.org/10.1109/AINAW.2007.336

[8] Pojda, J., Wolff, A., Sbeiti, M. and Wietfeld, C. (2011) Performance Analysis of Mesh Routing Protocols for Uav Swarming Applications. 8th International Symposium on Wireless Communication Systems (ISWCS), Niagara Falls, 21-23 May 2007, 317-321. https://doi.org/10.1109/ISWCS.2011.6125375

[9] Yang, H., Luo, H., Ye, F., Lu, S. and Zhang, L. (2004) Security in Mobile Ad Hoc Networks: Challenges and Solutions. IEEE Wireless Communications, 11, 38-47. https://doi.org/10.1109/MWC.2004.1269716

[10] Garg, N. and Mahapatra, R. (2009) Manet Security Issues. IJCSNS, 9, 241. 